\title{
The Effects of the Film Thickness and Roughness in the Anodization Process of Very Thin Aluminum Films
}

\author{
Ottone $\mathrm{C}^{1,2}$, Laurenti $\mathrm{M}^{1,2}$, Bejtka $\mathrm{K}^{1}$, Sanginario $\mathrm{A}^{1,2}$ and Cauda $\mathrm{V}^{* 1}$ \\ ${ }^{1}$ Center for Space Human Robotics@Polito, Istituto Italiano di Tecnologia, Corso Trento, 21, Turin, Italy \\ ${ }^{2}$ Politecnico di Torino, Dipartimento di Scienza Applicata e Tecnologia (DISAT), Corso Duca Degli Abruzzi, 24, \\ Turin, Italy.
} *Corresponding author: Cauda V, Center for Space Human Robotics@Polito, Istituto Italiano di Tecnologia,
Corso Trento, 21, Turin, Italy, Fax: 011090 3401, Tel: 011090 3436, E-mail: valentina.cauda@iit.it

Citation: Ottone C, Laurenti M, Bejtka K, Sanginario A, Cauda V (2014) The Effects of the Film Thickness and Roughness in the Anodization Process of Very Thin Aluminum Films. J Mater Sci Nanotechnol 1(1): S107. doi: 10.15744/2348-9812.1.S107

Received Date: May 28, 2014 Accepted Date: May 30, 2014 Published Date: July 18, 2014

\begin{abstract}
The anodization of aluminum foils having micrometer thickness is a common process and results in hexagonally self-ordered alumina membranes. However, anodic aluminum oxide (AAO) membranes fabricated from nanometer-thin films present new challenges to the anodization process, since aluminum films adheres poorly on supporting substrates and the smoothness of the film is highly related to the kind of substrate. In the current work we studied the effect of the aluminum thickness and roughness, using films ranging from 100 to $800 \mathrm{~nm}$ in thickness and from 2 to $15 \mathrm{~nm}$ in root means square roughness (on a scan area of $100 \mu \mathrm{m}^{2}$ ), on the final alumina morphology. We deposited Al thin films by sputtering method on transparent conductive glass substrates. A strong dependence between the Al film roughness and the final alumina pore organization was observed. It was also determined that by reducing the $\mathrm{Al}$ film thickness, smaller Al grains were generated, leading to a homogenous pore formation. It was found that, for thicknesses below 300 $\mathrm{nm}$, the electrolyte used to perform anodization becomes a critical parameter due to the competitive effect of aluminum delamination with respect to the anodization reaction. Phosphoric acid showed less delamination problems than oxalic acid.

Keywords: Anodic aluminum oxides; Thin films anodization; Hexagonal symmetry; Delamination; Nanometer thickness
\end{abstract}

\section{Introduction}

The oxidation of aluminum (Al), under controlled electrochemical process, leads to the formation of a self-organized array of parallel cylindrical nanopores perpendicular to the top of the underlying Al substrates. This structure is denoted as anodic aluminum oxide (AAO). This bottom-up technique offers many advantages since it is a cost effective, large scalable method and yields to porous membranes with nanoscale precision.

AAO membranes have been extensively used as a template in the fabrication of nanostructured materials [1-3], providing a lowcost alternative to lithography. A common example is the fabrication of metal [4,5] and metal oxides nanowires [6,7] that can be applied to Li-ion batteries, energy storage, solar cells, resistors, transistors and nanoreactors. Other considerable potential fields of applications are using AAO as catalytic and molecular separation membranes; and as drug or gene delivery carrier [8].

Hexagonally ordered patterns of alumina nanopores can be easily obtained in the reaction when starting from Al foils [9,10]. Many studies have focused on the comprehension on how parameters like electrolyte type, voltage and temperature affect the pore formation of bulk aluminum anodization [11-13]. However, reducing the thickness of Al down to nanometer scale changes the dependence to these parameters, generating a new interest in the optimization of the synthesis conditions.

Thick AAO membranes are typically prepared using the so-called two-step anodization method, in which the Al foil undergoes a first anodization with the only scope of matching the surface. This step yields to irregular alumina surfaces, i.e. irregular pore diameter with random distribution at the top and hexagonally patterned at the bottom [14]. Then, this alumina is dissolved, molding a pattern of hexagonally arranged depressions on the aluminum surface. In the second anodization step, these depressions act as nucleation sites for the forming alumina.

By scaling down towards thin films, the sacrificial alumina of the first anodization step would represent a high percentage of the starting Al film. Therefore only one anodization step is applied and the nanometer-thick AAO membranes morphology is thus strongly connected to the quality of the deposited aluminum film. Additionally thin membranes show poorly organized nanopores, including different diameter and hexagonal cell sizes, variable interpore distances and irregular arrangement. Recent works have demonstrated the application of image analysis tools for quantification of the resulting uniformity of the pore distribution [15,16]. 
Thus, fabricating self-ordered AAO membranes starting from thin Al films represents a challenge in nanotechnology. The fabrication of such thin AAO membranes requires the deposition of aluminum film, for example by the radio-frequency (RF) magnetron sputtering technique. It is a physical vapor deposition method, where an inert gas (usually argon) is ionized to create the plasma used for the extraction of atoms from a solid source (target). Sputtering allows the deposition of very thin, highquality films, on different kind of large-area substrates like silicon, transparent conductive oxide coated glasses and metal foils. If compared to the evaporation method, which is the other physical vapor deposition technique widely used for the deposition of $\mathrm{Al}$ films, sputtering shows the advantage of being a room temperature, plasma-assisted process. The final quality of sputtered Al films strictly depends on the working conditions. In particular, gas flows and pressures, the deposition rate and the substrate temperature strongly affect the surface morphology and crystal structure of the resulting $\mathrm{Al}$ films. By properly varying one or more of these parameters, uniform, smooth and highly crystalline Al films can be obtained. Therefore the deposition process is one of the key parameters to be finely controlled in order to obtain high quality and ordered AAO thin membranes.

It is also necessary to properly select the supporting substrates on which the Al film is deposited, in order to obtain a good mechanical stability. The choice of the support depends on the final application of the AAO membrane, and can range from rigid conductive substrates, such as transparent conductive oxide coated glasses [17-19], to silicon wafers [20,21] to flexible polymers [22]. The use of conductive substrates has the advantage of ensure the electric contact with $\mathrm{Al}$ until its complete anodization. Otherwise an irregular consumption of $\mathrm{Al}$ can occur, resulting in a loss of the contact with the power supply. However also insulating substrates can be used, such as glass or $\mathrm{SiO}_{2}$ coated silicon wafers, as previously reported [23,24]. In these cases, depending on the anodization cell characteristics, the final product can still contain non-anodized Al.

One problem associated to the anodization of thin aluminum films is the risk of delamination of the deposited metal from the supporting substrate [25]. The delamination occurs because of gas evolution due to local high current densities developed through the thin $\mathrm{Al}$ film. It was also reported that significant Joule heating can occur at the pore bottom because of such high current densities [26]. A reported solution for Indium Tin Oxide (ITO) coated glasses consists in the use of an adhesive layer having a higher affinity to the substrate than to aluminum: metals such as Ti or W have shown good performances [17].

This work reports on the anodization process of nanometer-thin Al films with a thickness ranging from 100 to $800 \mathrm{~nm}$ on ITOcoated glass substrates. In particular, we study the challenges due to the $\mathrm{Al}$ deposition process and the supporting substrate. We focus on the starting $\mathrm{Al}$ film roughness and its effect on the final distribution of the alumina pores. We also analyze the differences of the anodization process by varying the starting $\mathrm{Al} \mathrm{film} \mathrm{thickness.} \mathrm{In} \mathrm{addition,} \mathrm{we} \mathrm{study} \mathrm{the} \mathrm{role} \mathrm{of} \mathrm{both} \mathrm{oxalic} \mathrm{and} \mathrm{phosphoric}$ acids as electrolyte in modifying the morphology of the final alumina membrane depending on the thickness and their role in the delamination process. In order to vary the final pore size, we also analyze the effect of time in the pore widening process of the AAO films in phosphoric acid.

The idea of performing the anodization on a conductive substrate, as ITO in this case, aims to maintain a conductive substrate with a bottom accessible electrode, in order to further use the AAO film as template for the growth of nanowires [27-29] and then their exploitation for sensing measurements requiring bottom and top electrodes.

\section{Materials and Methods}

\section{Aluminum film}

Commercial ITO-coated glass (Kintech; $10 \Omega / \mathrm{sq}$ ), cut in pieces of $1.5 \mathrm{~cm} \mathrm{x} 2.5 \mathrm{~cm}$, were used as conductive substrates. The slides were sonicated for 10 min each in acetone (99.5\%, Sigma Aldrich) and 2-propanol (99.5\%, Sigma Aldrich), dried with nitrogen and subjected to oxygen plasma cleaning for 10 minutes.

$\mathrm{Al}$ films were deposited onto ITO glass by the RF magnetron sputtering technique, starting from a 4" in diameter Al target (GoodFellow, 99.999\% purity). Before starting the depositions, in order to prevent the presence of any contaminants and pollutants, suitable vacuum conditions were obtained with a rotary and a turbomolecular pump, and base pressure values around $2.6 \bullet 10^{-5} \mathrm{~Pa}$ were achieved. Suitable deposition parameters, summarized in Table 1, were selected in order to obtain Al thin films with different thicknesses and surface roughness, with the aim of comparing the influence of these aspects on the anodization process. Before each deposition, the target was cleaned with a 60 min sputtering process in a pure Ar atmosphere, with the aim to prevent any incorporation of contaminants in the films and to remove the native oxide which is present on the surface of the Al target. All the depositions were carried out at room temperature, in a pure Ar atmosphere at a working pressure of $0.7 \mathrm{~Pa}$, with a target-tosubstrate distance of about $8 \mathrm{~cm}$. A RF voltage at a working frequency of $13.56 \mathrm{MHz}$ was employed to create the plasma.

\section{$\mathrm{Al}$ anodization}

Aluminum films were anodized with $0.3 \mathrm{M}$ oxalic acid at $40 \mathrm{~V}$ and $20 \mathrm{mM}(10 \% \mathrm{wt}$.) phosphoric acid at $60 \mathrm{~V}$. The temperature was varied from 0 to $5{ }^{\circ} \mathrm{C}$ [30]. A thin layer of crystal bond was applied to the edges of the sputtered $\mathrm{Al}$ film to prevent the contact of the ITO layer with the electrolyte and thus avoid the undesired ITO etching. The anodization current was monitored using the LabVIEW software and the anodization process was stopped when the Al film became transparent and the current density reached a plateau. The different anodization processes were repeated three times per each sort of sample. With the scope of having larger AAO channels, the samples were also immersed in 5\% wt. phosphoric acid for 20-40 min at RT. 


\begin{tabular}{|c|c|c|c|c|c|}
\hline Sample name & RF power [Watt] & $\begin{array}{c}\text { Average thickness } \\
{[\mathbf{n m}]}\end{array}$ & Deposition rate [nm/min] & $\begin{array}{c}\text { RMS } \\
{[\mathrm{nm}]}\end{array}$ & $\begin{array}{c}\text { Average lateral size of Al grains } \\
{[\mathrm{nm}]}\end{array}$ \\
\hline A & 100 & 800 & 10 & 14.9 & $800^{\mathrm{b}}$ \\
\hline B & 30 & 800 & 3.3 & 4.5 & 300 \\
\hline C & 30 & 300 & 3.3 & 4.1 & 60 \\
\hline D & 30 & 100 & 3.3 & 2.0 & 45 \\
\hline
\end{tabular}

${ }^{\mathrm{R} M S}=$ Root Mean Square

'The sample is not uniform, thus the average size of the $\mathrm{Al}$ grains is about $800 \mathrm{~nm}$, but smaller grains ranging from 20 to $90 \mathrm{~nm}$ were also identified.

Table 1: Deposition conditions and surface characteristics of the sputtered aluminum thin films.

\section{Characterization}

The morphology was characterized by Field Emission Scanning Electron Microscopy (FE-SEM, ZEISS Dual-Beam Auriga). The side faces were prepared by first scratching the ITO-AAO samples on the glass backside with a diamond cutter, then by dipping in liquid $\mathrm{N}_{2}$ and thus by fracturing the sample into two pieces. Topographic images of the aluminium surface prior to anodization were obtained by Atomic Force Microscopy (AFM, A.P.E. Research A100 in contact mode (applied force: $5 \mathrm{nN}$ ) at different scan sizes.

\section{Results and Discussion}

Al thin films were deposited on conductive ITO-glass substrates by the RF magnetron sputtering technique. The used of ITOglass substrates allowed the complete anodization of the $\mathrm{Al}$ film. The influence of the sputtered Al films morphology on the pore organization of the AAO was analyzed. To this purpose, the effect of different deposition rates on the surface characteristics of the final Al films was investigated. Moreover, Al films deposited at the lower deposition rate, but with different thicknesses (ranging from $100 \mathrm{~nm}$ up to $800 \mathrm{~nm}$ ) were also compared. All the films were characterized by AFM topography, before the anodization process.

First, the influence of different deposition rates on the surface morphology of the final film was considered. Samples A and B, as reported in Table 1, refer to Al films having the same average thickness $(800 \mathrm{~nm})$, but deposited at different deposition rates (10 $\mathrm{nm} / \mathrm{min}$ and $3.3 \mathrm{~nm} / \mathrm{min}$ respectively). AFM topography of Sample A is reported in Figure 1a and shows the presence of great asperities (hillocks) and large irregular grains. The surface morphology of Sample B is shown in Figure 1b and a smooth surface, with regular grains can be observed. The root mean square (RMS) roughness of both samples are reported in Table 1, showing a consistently lower value $(4.5 \mathrm{~nm})$ for Sample B with respect to Sample A $(14.9 \mathrm{~nm})$. The AFM topography (Figure S-1 in the Supporting Information) and roughness $(1.6 \mathrm{~nm}$ ) of the ITO substrate were also measured, showing almost no influence of the substrate on the morphology of the deposited Al. Indeed, the characteristics of the surface morphology are strictly related to the deposition rate used during the sputter deposition [35] and films with rough surfaces and poor crystal-quality are expected for higher deposition rates [35]. The deposition rate strongly affects the processes of surface diffusion of atoms, nucleation and coalescence of the metal clusters during thin-film growth [36]. At low deposition rates the number of $\mathrm{Al}$ atoms arriving onto the substrate surface is low, and the formation of $\mathrm{Al}$ nuclei is not favored. This implies a low nucleation density, which results in a surface with small grains (i.e. $300 \mathrm{~nm}$, Table 1). When the deposition rate is high, the number of Al atoms arriving on the surface is high, resulting in bigger nuclei formation and bigger grains that can be grown $(800 \mathrm{~nm})$. In this work, a high and low deposition rate $(10 \mathrm{~nm} / \mathrm{min}$ and $3.3 \mathrm{~nm} / \mathrm{min}$ ) were obtained by using the highest and lowest RF power values (100 W and $30 \mathrm{~W}$ respectively). Therefore, films with higher roughness and non-uniform grains, as reported for Sample A in Figure 1a, were achieved when the highest deposition rate was used. On the contrary, a smooth surface with uniform grains was observed in the case of Sample B in Figure $1 \mathrm{~b}$, which was deposited with the lowest deposition rate. From the comparison of AFM topography and surface roughness profile of Samples A and B it can be concluded that using a lower deposition rate is the best condition for obtaining smooth Al films with uniform and regular grains sizes. Once the most suitable surface morphology conditions were selected, the influence of different thicknesses in Al films on the anodization process was investigated. Samples C and D refer to Al films with an average thickness of $300 \mathrm{~nm}$ and $100 \mathrm{~nm}$, respectively. These were all deposited with an average deposition rate of $3.3 \mathrm{~nm} / \mathrm{min}$, which is the lowest value used in this work, as stated before. Al films being $300 \mathrm{~nm}$-thin (Figure 1c) and $100 \mathrm{~nm}$-thin (Figure 1d) have similar morphologies, with a smooth surface (RMS roughness of 4.1 and $2.0 \mathrm{~nm}$ respectively) and an average grain size of 60 and $45 \mathrm{~nm}$, respectively (Table 1). According to the literature [37,38], here the surface roughness of Al films decreases by decreasing the film thickness. When comparing the surface morphology of Sample B with Samples C and D, it can be highlighted that reducing the film thickness from $800 \mathrm{~nm}$ to $300 \mathrm{~nm}$ or less resulted in films with smoother surfaces and smaller grains. 

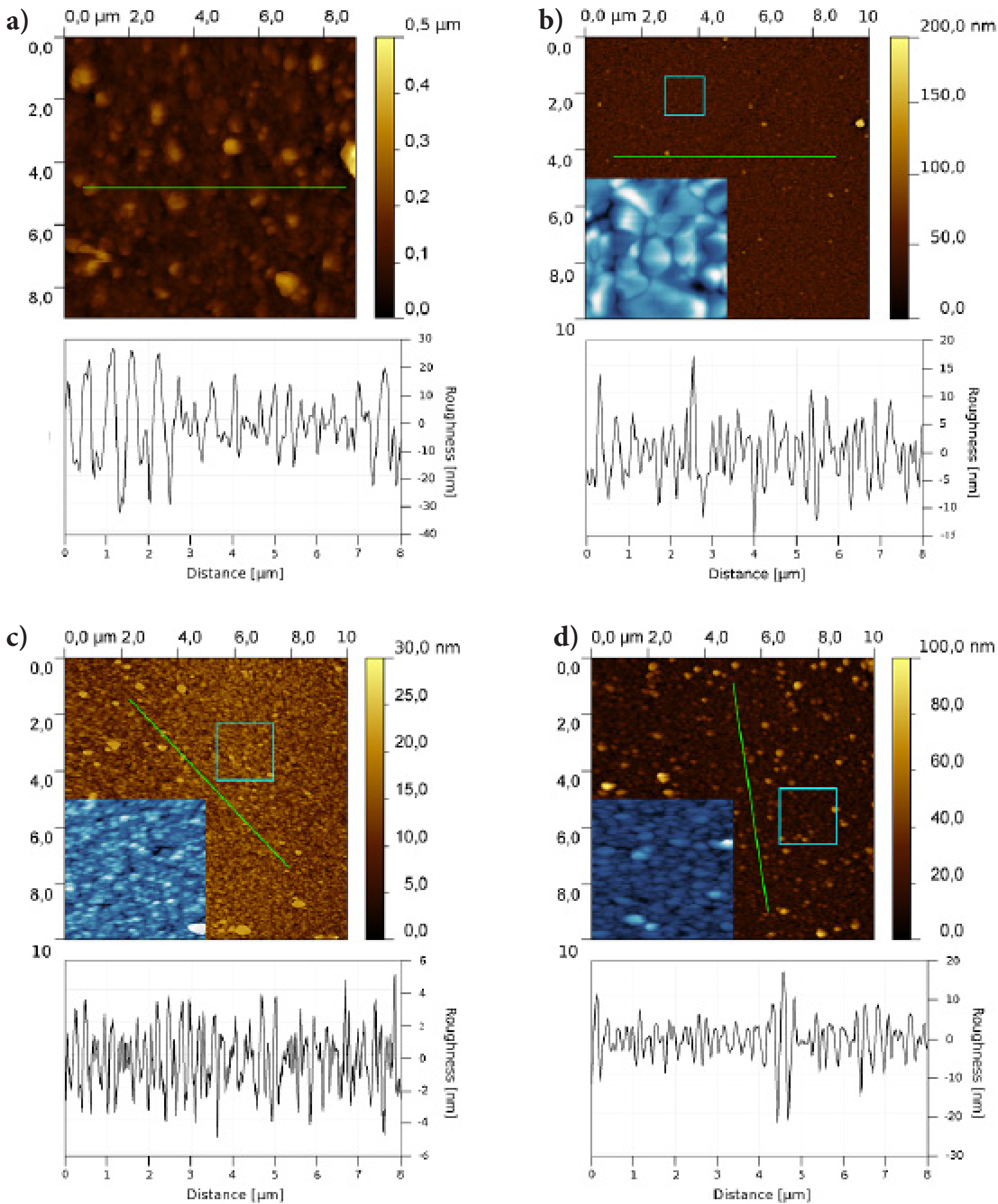

Figure 1: AFM topographic scan of a $100 \mu \mathrm{m}^{2}$ area of (a) $800 \mathrm{~nm}$ thin Al film sputtered with a deposition rate of $10 \mathrm{~nm} / \mathrm{min}$ (Sample A); (b) $800 \mathrm{~nm}$ (Sample B), (c) $300 \mathrm{~nm}$ (Sample C), (d) $100 \mathrm{~nm}$ (Sample D) thin aluminum film sputtered with a deposition rate of $3.3 \mathrm{~nm} / \mathrm{min}$. The inset images show a magnified scan area of $2.25 \mu \mathrm{m}^{2}$. Sample A has no inset because the magnification area is comparable with the grain lateral size (about $800 \mathrm{~nm}$ ). The surface roughness profile (collected from the green line depicted in the AFM topography) is reported below each topographic image. 
Figure 2 shows the most representative top and cross-sectional views of the AAO membranes obtained after the anodization of the $800 \mathrm{~nm}$-thick Al films prepared with both deposition rates (3.3 and $10 \mathrm{~nm} / \mathrm{min}$ ). Sample A yielded to regularly arranged pores only on the small rectangular flat regions (Figure $2 \mathrm{a}$ ). Those regions are isolated and do not form a continuous film. Pores are also formed on the rest of the sample but with random distribution and irregular sizes. From the cross-sectional view in Figure $2 \mathrm{~b}$, the pores do not show a straight and parallel pattern penetrating into the membrane, but are instead bent and randomly oriented, following the inhomogeneous grain shape.
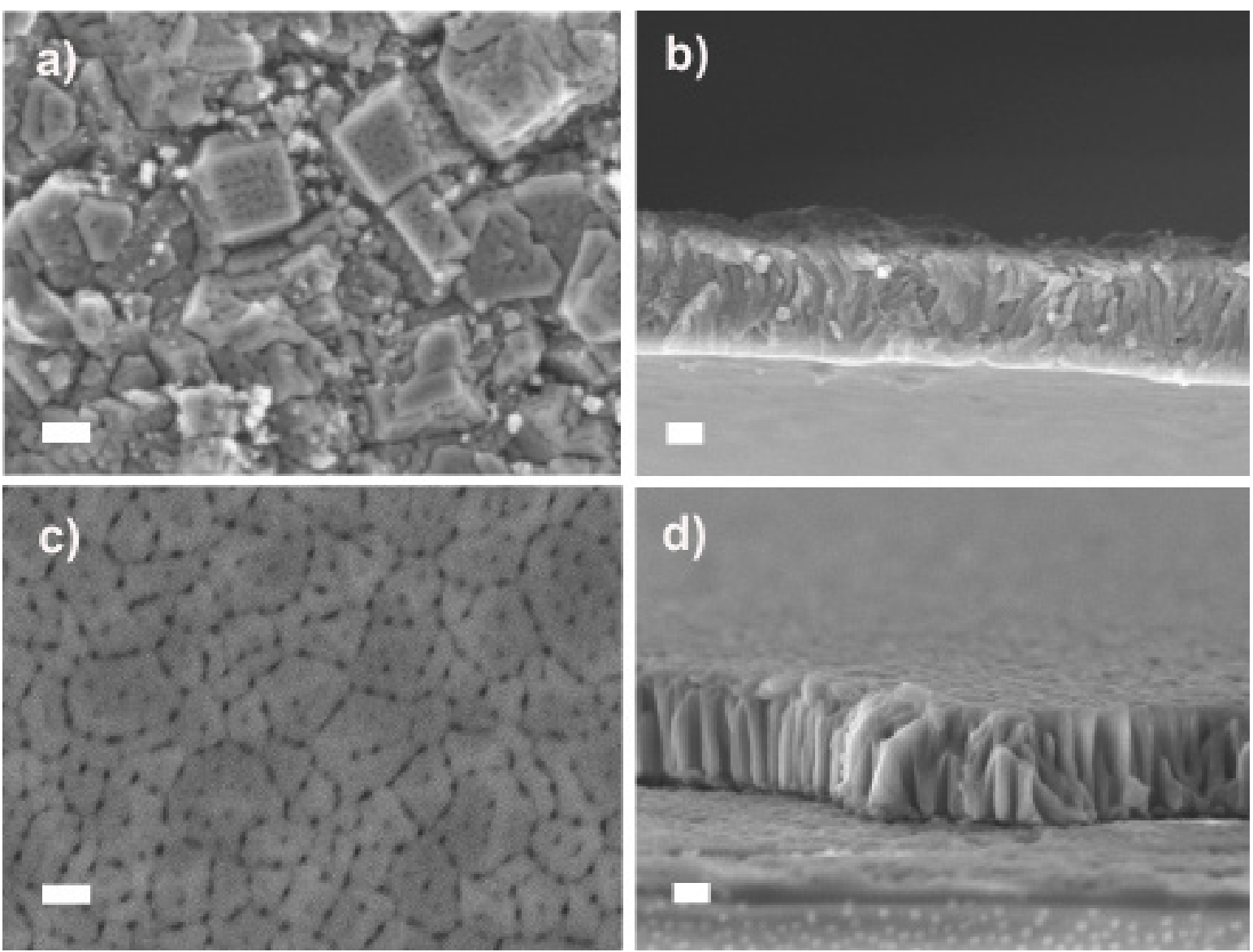

Figure 2: FE-SEM image of AAO membrane obtained on ITO-coated glass slide after anodization of $800 \mathrm{~nm}$ Al-film in oxalic acid (0.3 M) at $40 \mathrm{~V}$. Al films prepared with a rate of $10 \mathrm{~nm} / \mathrm{min}$ : (a) top and (b) cross section views; and with a rate of $3.3 \mathrm{~nm} / \mathrm{min}$ : (c) top and (d) cross-section views. Scale-bar are all set to $200 \mathrm{~nm}$.

In contrast, the good quality of the $\mathrm{Al}$ films prepared with a lower deposition rate $(3.3 \mathrm{~nm} / \mathrm{min}$, i.e. Sample B) is reflected in an AAO membrane with a uniform pore organization. This regular anodization on the surface is attributed to a constant current density because the grains are in contact with the electrolyte only from the upper face, and electrolyte cannot penetrate into the grain boundaries. This also reduces greatly the delamination risk. The former Al grain boundaries are still easily recognizable: the pores grew-up preferentially on the grain boundaries ( $300 \mathrm{~nm}$ in size, calculated from the AFM images in Figure 1b), showing a larger diameter $(20 \mathrm{~nm})$ in comparison to the in-grain pores $(8 \mathrm{~nm})$. From the cross-sectional view in Figure $2 \mathrm{~d}$ it can be observed that all the pores are parallel to each other. The comparison between the cross section images of the AAO membrane prepared by using the highest and lowest deposition rates are shown in Figures $2 \mathrm{~b}$ and $2 \mathrm{~d}$, respectively, confirming that the lowest deposition rate of $3.3 \mathrm{~nm} / \mathrm{min}$ yields to well organized and ordered AAO membranes, having pores distributed parallel to each other and perpendicular to the ITO substrate.

The further films were then fabricated following the deposition rate of $3.3 \mathrm{~nm} / \mathrm{min}$ and only the deposition time was modified in order to obtain different Al thicknesses (Table 1). Figure 3 shows the surface structure of 300 and 100 nm-thick AAO membranes. Figures $3 \mathrm{a}$ and $3 \mathrm{~b}$ show the FE-SEM image of the as-made $300 \mathrm{~nm}$-thick AAO fabricated with both oxalic and phosphoric acids, respectively. The membrane prepared with the phosphoric acid has in average larger pores $(60 \mathrm{~nm})$ and cell sizes (120 nm) with respect to the ones made with oxalic acid $(20 \mathrm{~nm}$ and $90 \mathrm{~nm}$, respectively). These results are also in line with the literature [17,25,30]. The AAO channels in these two cases are very similar: the pore morphology is homogeneous throughout the whole surface of the samples, having a different morphology in comparison with the $800 \mathrm{~nm}$-thick membrane reported in Figure 2, and demonstrating that the final pore feature does not depend on the electrolyte but on the thickness, due to its correlation with the roughness in the AAO resulting from thin Al films. 

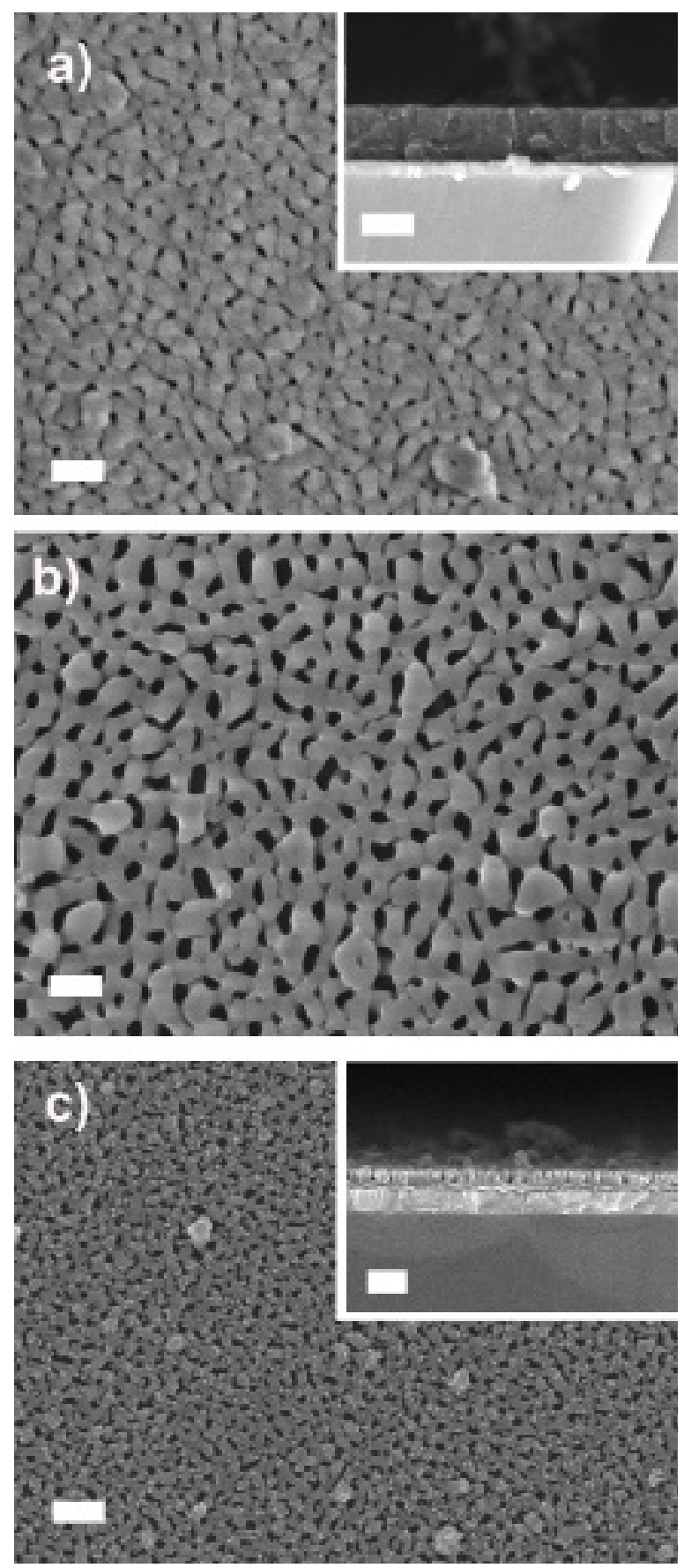

Figure 3: FE-SEM images of AAO membranes obtained after anodization in (a) oxalic acid of $300 \mathrm{~nm}$ film (Inset: cross-sectional view); and in phosphoric acid of (b) $300 \mathrm{~nm}$ film and (c) $100 \mathrm{~nm}$ film in top view (Inset: cross-sectional view). Scale-bars are all set to $200 \mathrm{~nm}$.

The electrolyte plays an important role during the anodization process, as it is able to sustain a significant flow of $\mathrm{Al}^{3+}$ ions from the metal substrate into the electrolyte. Thompson et al. reported that the loss of $\mathrm{Al}^{3+}$ ions from the metal substrate is governed by two mechanisms: (i) the direct expulsion of ions by the applied electric field and (ii) the dissolution of the forming oxide layer. In the regions of high current flows under an applied electric field, the dissolution rate increases [39]. Experimentally, we measured that the anodization, until obtaining transparent films, takes longer times when using phosphoric acid with respect to the anodization in oxalic acid. This indicates that the $\mathrm{Al}$ dissolution in the longitudinal direction is higher with oxalic acid and slower with phosphoric acid. Thus, oxalic acid yields smaller pores, as indicated before, whereas phosphoric acid first removes $\mathrm{Al}^{3+}$ ions in the transversal direction faster than the oxalic acid, generating larger pores. This finding seems to be in contrast with what reported by Toccafondi et al. [16], where the phosphoric acid shows the faster anodization rate with respect to the oxalic one. However, this is related to the different phosphoric acid concentration: we used a concentration which is twenty time lower than that used in the reported paper $(0.4 \mathrm{M})$. 
Journal of Materials Science \& Nanotechnology

Phosphoric acid was then chosen for preparing $100 \mathrm{~nm}$-thin AAO membranes. It was observed that performing the anodization of very thin $(100 \mathrm{~nm})$ aluminum films was not possible with oxalic acid because the film delaminated from the ITO substrate rapidly. The anodization of $100 \mathrm{~nm}$-thin Al films with use of the phosphoric acid yielded to AAO pore formation at the grain boundaries, shown in a top view FE-SEM image (Figure 3c). The behavior is similar to that of the $300 \mathrm{~nm}$-thin sample. The pores do not have a circular shape at the top, but they follow the shape of the grain boundaries. The inset of Figure $3 \mathrm{c}$ shows the cross-section view of this sample and reveals that the pores are all columnar.

As reported above for the $800 \mathrm{~nm}$-thin sample, the pores were formed at the grain boundaries. However, in case of the $300 \mathrm{~nm}$ and $100 \mathrm{~nm}$-thin membranes from Samples C and D respectively, the presence of smaller Al grains of 60 and $45 \mathrm{~nm}$ in each case, respectively, creates almost a continuous network of pores, where grain boundaries are hardly recognizable, as shown in Figures $3 \mathrm{~b}$ and c. It has to be noted that hexagonally ordered symmetry of the AAO pores is not obtained in any sample.

In order to both study the adhesion of the alumina layer and obtain larger pores, the AAO membranes were subjected to pore widening, i.e. a chemical attack with $5 \%$ wt phosphoric acid for a time up to 80 minutes. All $800 \mathrm{~nm}$ - and $300 \mathrm{~nm}$-thin samples showed a good adhesion to the substrate throughout the entire supporting surface.
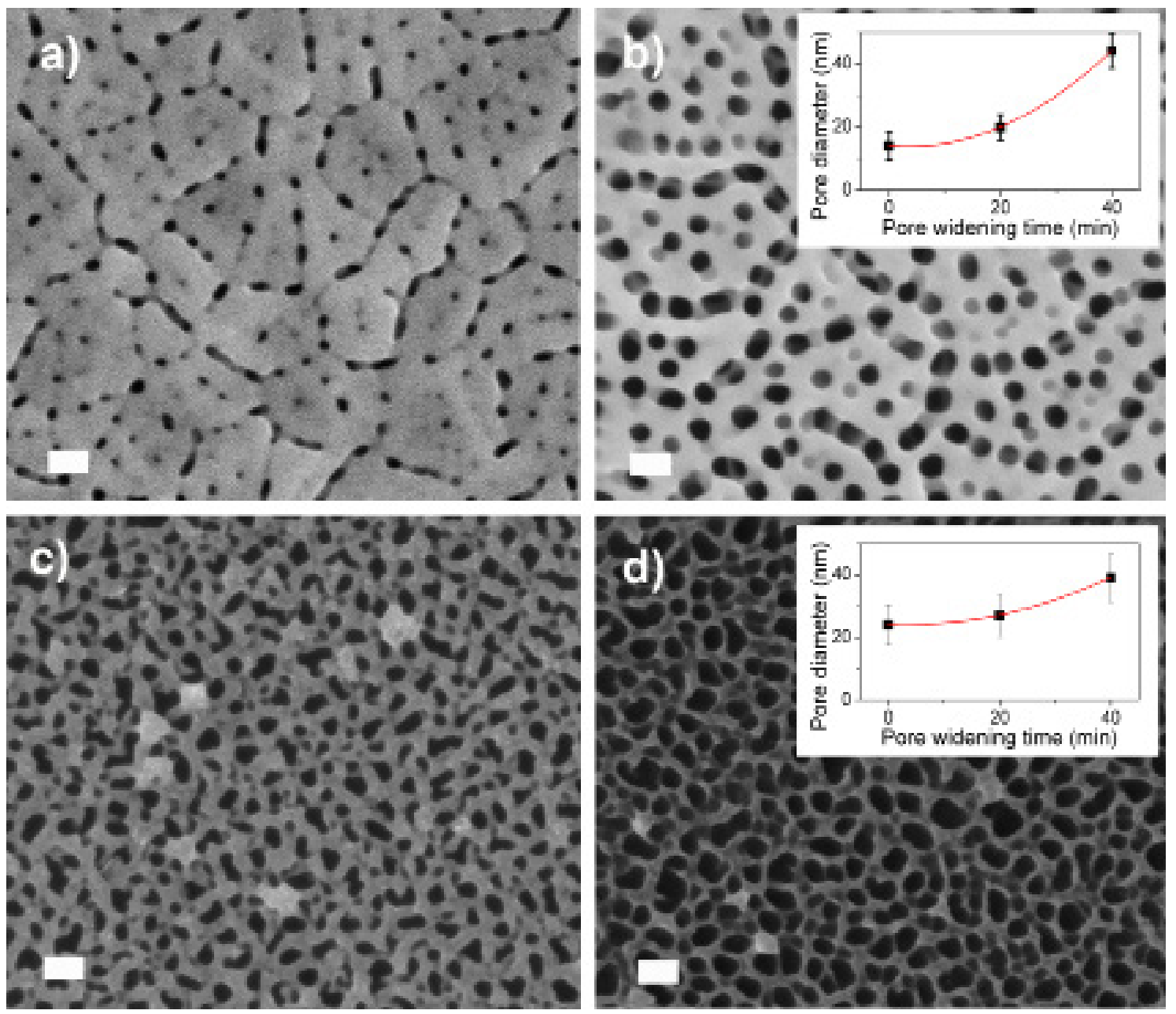

Figure 4: FE-SEM image of AAO membrane obtained from $800 \mathrm{~nm}$ film (a) after pore widening in phosphoric acid (5\%) for 20 minutes and (b) 40 minutes. AAO membrane obtained from $100 \mathrm{~nm}$-thin film after (c) 20 minutes and (d) 40 minutes of pore widening. Scale-bar are all set to $100 \mathrm{~nm}$. Insets of Figures b) and d) both show the plot of the pore diameter versus widening time. The red line is a guide for the eyes.

Pore widening helps indeed to obtain uniform pore size both at grain boundaries and inside the grains. Figures $4 \mathrm{a}$ and $4 \mathrm{~b}$ show the FE-SEM images of the $800 \mathrm{~nm}$-thin membranes after $20 \mathrm{~min}$ and $40 \mathrm{~min}$ process in phosphoric acid, respectively. The chemical attack gradually enlarged both the pores at the grain boundaries and inside the grains, but with different rates. Indeed the pores situated inside the grain enlarge faster with respect to the pores placed at the boundaries. However, after 40 min process, pores with uniform diameter (about $42 \mathrm{~nm}$ ) were obtained, resulting in a AAO membrane with homogenous pore size. In contrast to the $800 \mathrm{~nm}$-thin AAO, the as made $100 \mathrm{~nm}$-thin samples showed larger pores ( $24 \mathrm{~nm}$ in diameter), after 20 minutes of pore widening the average pore diameter slightly increased up to $27 \mathrm{~nm}$, but after 40 minutes the pores enlarged up to about $60 \%$ (40 nm) of the initial size, as depicted in Figure $4 \mathrm{c}$ and $4 \mathrm{~d}$, respectively. In our studies we did not observed a linear relationship between the porewidening time and the average nanopore diameter (see the insets of Figure 4), as in contrast reported by Musselman et al. [40] of $\mu \mathrm{m}$-thick AAO membranes. This discrepancy is attributed to the irregular pore diameter of our samples and the great influence of 
both the $\mathrm{Al}$ grain size and the film thickness on the pore formation. The overall widening process is not linear due to the different pore widening rates between the pores situated inside the grain and those placed at the boundaries.

It is worth noting that the $100 \mathrm{~nm}$-thin AAO membranes can sustain long times of pore widening despite the low thicknesses. Indeed, after 80 minutes we observed regions where the alumina was dissolved and regions where the pores are still present, as reported in the Figure S-2 of the S.I.

\section{Conclusion}

In the present paper we have established the importance of the $\mathrm{Al}$ smoothness and thickness in nanometric-thin films for reaching well ordered pore arrangement of AAO membranes. Regarding the study of the Al thickness, we observed that the final AAO membrane morphology always maintained the original shape of the starting Al thin film, still showing the $\mathrm{Al}$ grain boundaries. In addition, we have identified a limitation in the anodization with oxalic acids for ultra thin Al films: below the thickness of 300 $\mathrm{nm}$, we observed that only phosphoric acid can lead to homogenously sized and parallel oriented AAO pores. In contrast heavy delamination problems are encountered when using oxalic acid.

The pore widening was applied as an additional step allowing to have regular pore sizes when the anodization has different nucleation preferences, i.e. at the grain boundaries or inside the grain. Homogeneous pore sizes were achieved at the end of 40 minutes pore widening, without affecting the AAO porous morphology.

The anodization study of these nanometer-sized Al films has brought new insight on the formation of very thin AAO membranes, which can then find potential applications as molecular separation membranes and as templates for one-dimensional nanostructure fabrication.

\section{References}

1. Hurst SJ, Payne EK, Qin L, Mirkin CA (2006) Multisegmented one-dimensional nanorods prepared by hard-template synthetic methods. Angew Chem Int Ed Engl 45: 2672-92.

2. Lai M, Riley DJ (2008) Templated electrosynthesis of nanomaterials and porous structures. J Colloid Interface Sci 323: 203-12.

3. Cauda V, Stassi S, Bejtka K, Canavese G (2013) Nanoconfinement: an effective way to enhance PVDF piezoelectric properties. ACS Appl Mater Interfaces 5: 6430-7.

4. Wirtz M, Yu S, Martin CR (2002) Template synthesized gold nanotube membranes for chemical separations and sensing. Analyst 127: 871-9.

5. Mu C, Zhang JP, Xu D (2010) Au nanoparticle arrays with tunable particle gaps by template-assisted electroless deposition for high performance surfaceenhanced Raman scattering. Nanotechnology 21: 015604.

6. Bae C, Yoo H, Kim S, Lee K, Kim J, et al. (2008) Template-directed synthesis of oxide nanotubes: fabrication, characterization, and applications. Chem Mater 20: $756-67$.

7. Ottone C, Bejtka K, Chiodoni A, Farias V, Roppolo I, et al. (2014) Comprehensive study of the templating effect on the ZnO nanostructure formation within porous hard membranes. New J Chem 38: 2058-65.

8. Fu J, Mao P, Han J (2008) Artificial molecular sieves and filters: a new paradigm for biomolecule separation. Trends Biotechnol 26: 311-20.

9. Nielsch K, Choi J, Schwirn K, Wehrspohn RB, Gösele U (2002) Self-ordering regimes of porous alumina: the 10 porosity rule. Nano letters 2: 677-80.

10. Lee K, Tang Y, Ouyang M (2008) Self-ordered, controlled structure nanoporous membranes using constant current anodization. Nano Lett 8: 4624-9.

11. Furneaux R, Rigby W, Davidson A (1989) The formation of controlled-porosity membranes from anodically oxidized aluminium. Nature 337: 147-9.

12. Sulka GD, Stępniowski WJ (2009) Structural features of self-organized nanopore arrays formed by anodization of aluminum in oxalic acid at relatively high temperatures. Electrochimica Acta 54: 3683-91.

13. Sun C, Luo J, Wu L, Zhang J (2010) Self-ordered anodic alumina with continuously tunable pore intervals from 410 to $530 \mathrm{~nm}$. ACS Appl Mater Interfaces 2: 1299-302.

14. Masuda H, Fukuda K (1995) Ordered metal nanohole arrays made by a two-step replication of honeycomb structures of anodic alumina. Science 268: 1466-8.

15. Stępniowski WJ, Michalska-Domańska M, Norek M, Czujko T (2014) Fast Fourier transform based arrangement analysis of poorly organized alumina nanopores formed via self-organized anodization in chromic acid. Mater Lett 117: 69-73.

16. Toccafondi C, Stępniowski WJ, Leoncini M, Salerno M (2014) Advanced morphological analysis of patterns of thin anodic porous alumina. Mater Charact 94: 26-36.

17. Chu SZ, Wada K, Inoue S, Todoroki S (2003) Fabrication and characteristics of nanostructures on glass by Al anodization and electrodeposition. Electrochimica Acta 48: 3147-53.

18. Chen W, Wu JS, Xia XH (2008) Porous anodic alumina with continuously manipulated pore/cell size. ACS Nano 2: 959-65.

19. Poinern GEJ, Ali N, Fawcett D (2011) Progress in nano-engineered anodic aluminum oxide membrane development. Materials 4: 487-526.

20. Kokonou M, Nassiopoulou AG, Giannakopoulos KP (2005) Ultra-thin porous anodic alumina films with self-ordered cylindrical vertical pores on a p-type silicon substrate. Nanotechnology 16: 103-6.

21. Shen L, Ali M, Gu Z, Min B, Kim D, et al. (2013) Preparation of anodic aluminum oxide (AAO) nano-template on silicon and its application to onedimensional copper nano-pillar array formation. Korean J Chem Eng 30: 221-7.

22. Mardare AI, Kaltenbrunner M, Sariciftci NS, Bauer S, Hassel AW (2012) Ultra-thin anodic alumina capacitor films for plastic electronics. physica status solidi (a) 209: 813-18. 
23. Toccafondi C, Thorat S, La Rocca R, Scarpellini A, Salerno M, et al. (2014) Multifunctional substrates of thin porous alumina for cell biosensors. J Mater Sci Mater Med: [Epub ahead of print].

24. Rabin O, Herz PR, Lin YM, Akinwande AI, Cronin SB, et al. ( 2003) Formation of thick porous anodic alumina films ans nanowire arrays on silicon wafer and glass. Adv Funct Mater 13: 631-8.

25. Weickert J, Palumbiny C, Nedelcu M, Bein T, Schmidt-Mende L (2011) Controlled growth of $\mathrm{TiO}_{2}$ nanotubes on conducting glass. Chem Mater 23: 155-62. 26. Lee W, Ji R, Gösele U, Nielsch K (2006) Fast fabrication of long-range ordered porous alumina membranes by hard anodization. Nature Mater 5: $741-7$.

27. Cauda V, Torre B, Falqui A, Canavese G, Stassi S, et al. (2012) Confinement in oriented mesopores induces piezoelectric behavior of polymeric nanowires. Chem Mater 24: 4215-21.

28. Cauda V, Stassi S, Bejtka K, Canavese G (2013) Nanoconfinement: an effective way to enhance PVDF piezoelectric properties. ACS Appl Mater Interfaces 5: 6430-7.

29. Ottone C, Bejtka K, Chiodoni A, Farías V, Canavese G, et al. (2014) Comprehensive study of the templating effect on the ZnO nanostructure formation within porous hard membranes. New J Chem 38: 2058-65.

30. Weickert J, Palumbiny C, Nedelcu M, Bein T, Schmidt-Mende L (2011) Controlled growth of $\mathrm{TiO}_{2}$ nanotubes on conducting glass. Chem Mater 23 : 155-62.

31. Ono S, Saito M, Ishiguro M, Asoh H (2004) Controlling factor of self-ordering of anodic porous alumina. Journal of the Electrochemical Society 151: B473-8.

32. Ono S, Saito M, Asoh H (2005) Self-ordering of anodic porous alumina formed in organic acid electrolytes. Electrochimica Acta 51: 827-33.

33. Sulka GD (2008) Highly ordered anodic porous alumina formation by self-organized anodizing. Nanostructured materials in electrochemistry: 1-116.

34. Vrublevsky I, Chernyakova K, Bund A, Ispas A, Schmidt U (2012) Effect of anodizing voltage on the sorption of water molecules on porous alumina. Applied Surface Science 258: 5394-8.

35. Bordo K, RUBAHN HG (2012) Effect of deposition rate on structure and surface morphology of thin evaporated al films on dielectrics and semiconductors. Materials Science/Medziagotyra 18: 313.

36. Paul A, Wingbermühle J (2006) Surface morphology for ion-beam sputtered Al layer with varying sputtering conditions. Applied Surface Science 252: 8151-5. 37. Lita AE, Sanchez JE (1999) Characterization of surface structure in sputtered Al films: Correlation to microstructure evolution. Journal of Applied Physics 85: $876-82$.

38Yan S, De-Quan Y, Jing-Zhong C (1999) A morphology study of magnetron-sputtered al films by atomic force microscopy. Journal of Materials Science Letters 18: 407-9.

39. Thompson G, Furneaux R, Wood G, Richardson J, Goode J (1978) Nucleation and growth of porous anodic films on aluminium. Nature 272 : 433-5. 40. Musselman KP, Mulholland GJ, Robinson AP, Schmidt-Mende L, MacManus-Driscoll JL (2008) Low-Temperature synthesis of large-area, free-standing nanorod arrays on ito/glass and other conducting substrates. Advanced Materials 20: 4470-75

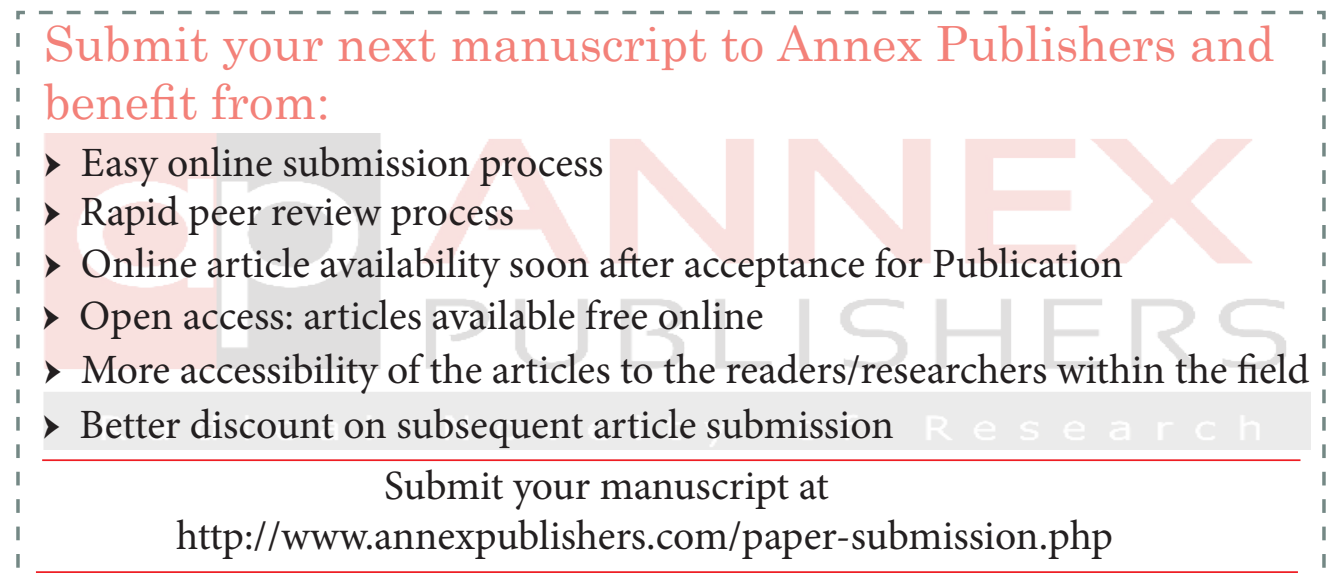

